\title{
ON THE RECORDING CODE WHICH IMPROVES THE GROOVE PARAMETER
}

\author{
K. TANAKA, H. MIZUNO and T. TAKADA
}

Teikyo University of Technology, Japan

\begin{abstract}
In order to increase the recording capacity the mark edge recording and $(1,7) \mathrm{RLL}$ code are employed in the second generation ISO/IEC optical disk standard. However this recording method degrades groove parameter at ROM area because $(1,7)$ RLL code has long Tmax which causes recording pattern of combination of long pits and short spaces. New $(2,5)$ RLL code whose Tmax is small were developed. Because Tmax/Tmin gets close to unity, the information capacity of the code is small so that detection window width becomes narrow. Because narrow band signal to noise ratio is large and jitter is small in RoM area, the window margin of $(2,5)$ RLL code written in ROM area is same as that of $(1,7)$ RLL code written in MO area when both codes are recorded in the same recording density. The window width of $(2,5) \mathrm{RLL}$ code is $0.4 \mathrm{~Tb}$ and Tmin is $1.2 \mathrm{~Tb}$ where $\mathrm{Tb}$ is one bit length of information bit.

KEYWORDS: RECORDING CODE, OPTICAL DISK, OPTICAL ROM, PRE-PIT
\end{abstract}

\section{INTRODUCTION}

In order to increase the recording capacity the mark edge recording and $(1,7) \mathrm{RLL}$ code are employed in the second generation ISO/IEC optical disk standard.[1] However, this recording method degrades groove parameter at ROM area where data is written in pre-pit form. It imposes difficulty on the drive design. This paper describes new recording code which improves groove parameter at the ROM area.

\section{IMPROVEMENT OF GROOVE PARAMETER}

When the reflected beam from land area decreases due to the existence of the pits in ROM area, groove parameters such as cross track signal (CTS) and push-pull signal are degraded so that optical head seek control becomes difficult. This is severe in the combination of the mark edge recording and $(1,7)$ RLL code. The worst case of it is the case where there is repetition of $7 \mathrm{~T}, 2 \mathrm{~T}$ pattern and $7 \mathrm{TS}$ correspond to the pits and 2Ts do to the spaces. Where ' $\mathrm{I}$ represents one channel bit length. The repetition of $8 \mathrm{~T}, 2 \mathrm{~T}$ dose not occur in present $(1,7)$ RLL code fortunately. Fig. 1 shows relationship between groove parameters and pit density.

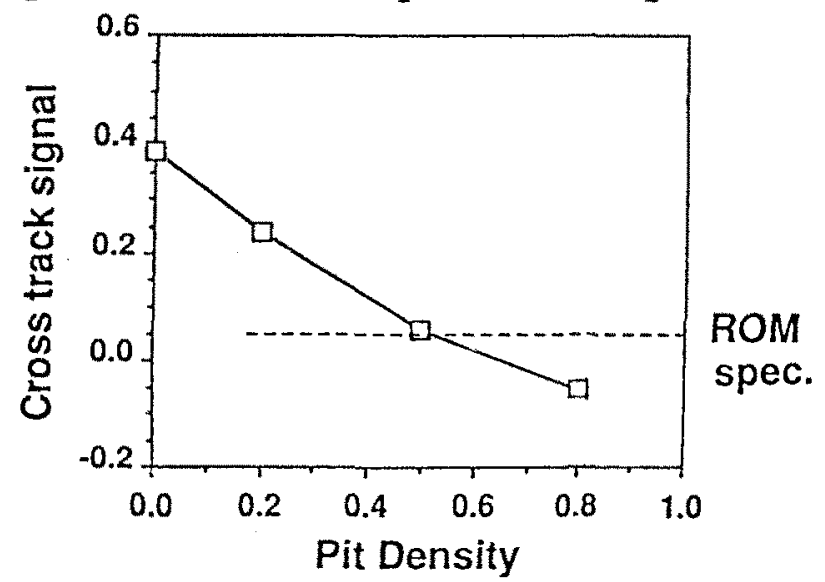

Fig.1: Relationship between groove parameters and pit density

The definition of pit density is the percentage of pit area in length on the land. Because the pit density of the worst case is 0.8 , the worst case CTS dose not satisfy the criteria 
specified by ISO/IEC. In order to solve this problem re-synch byte is used to control DSV(Digital Sum Variance) of channel bits so that long term average of the cross track signal barely meet the specification. However, there is still place where the CTS dose not meet the specification in short term average. It still gives burden to the seek control of drives.

One of the method to improve CTS is to employ mark position recording. However, employment of mark edge recording is preferable from the recording density point of view if it is possible. Most of the recording codes have been developed only to increase recording density. The code developed with giving consideration to both groove parameter and recording density can solve the problem.

When a code, whose Tmax is short and Tmin is long, is employed, the degradation of the groove parameter by the presence of pits can be eased. Because size of encoding and decoding circuitry of recording code are small compared with rest of whole circuit in optical disk drive, employment of recording code, different from that in MO area, in ROM area is acceptable.

\section{THE RECORDING CODE}

Coding/Decoding Circuit and Code Parameter

A couple of types of $(2,5)$ RLL codes, whose combination of Tmax and Tmin is better than that of $(1,7)$ RLL code, were developed and studied. Among them Fixed block type was selected. The conversion table is shown in Table 1.The code parameters are listed in Table 2 . Where Cap. stands for Shannon capacity of the code. $(2,5)$ RLL code can be coded using sliding block

Table1 : Encoding algorithm of fixed block $(2,5) R L L$ code

\begin{tabular}{|c|c|c|c|}
\hline \multirow{2}{*}{ Input bits } & \multicolumn{3}{|c|}{ Channel bits } \\
\cline { 2 - 4 } & \multicolumn{2}{|c|}{ * Preceding channel bits } \\
\hline 0000 & 0100010000 & $\leftarrow$ & 0000100100 \\
0001 & 0100001000 & $\leftarrow$ & 0001001001 \\
0010 & 0100010001 & $\leftarrow$ & 0000010001 \\
0011 & 1000100001 & $\rightarrow$ & 0000100001 \\
0100 & 1000010000 & $\rightarrow$ & 0001000100 \\
0101 & 1000001000 & $\rightarrow$ & 0001001000 \\
0110 & 0100010010 & $\leftarrow$ & 0000010010 \\
0111 & 0100100010 & $\rightarrow$ & 0001000001 \\
1000 & 1000100100 & $\rightarrow$ & 0010000100 \\
1001 & 1001001000 & $\rightarrow$ & 0010001000 \\
1010 & 1000010001 & $\rightarrow$ & 0010010001 \\
1011 & 1000100010 & $\rightarrow$ & 0000100010 \\
1100 & 1001000100 & $\rightarrow$ & 0010010000 \\
1101 & 0100001001 & $\rightarrow$ & 0010001001 \\
1110 & 1000010010 & $\rightarrow$ & 0010010010 \\
1111 & 1001000010 & $\rightarrow$ & 0001000010 \\
\hline
\end{tabular}

Table2: Code Parameter of $(2,5)$ RLL Code

\begin{tabular}{|c|c|c|c|}
\hline Item & Value & Item & Value \\
\hline$m$ & 2 & $T_{\min }$ & 1.2 \\
\hline$n$ & 5 & $T_{\max }$ & 2.4 \\
\hline$d$ & 2 & $D R$ & 1.2 \\
\hline$k$ & 5 & $D S V$ & $\infty$ \\
\hline$T \omega$ & 0.4 & Cap. & 0.465 \\
\hline
\end{tabular}

method as shown in Fig. 2. Coding algorithms is shown in Annex 1 . Decoding is the inverse process of encoding so that it can be done in similar way. Fig. 3 shows run length distribution when random data is encoded. When 


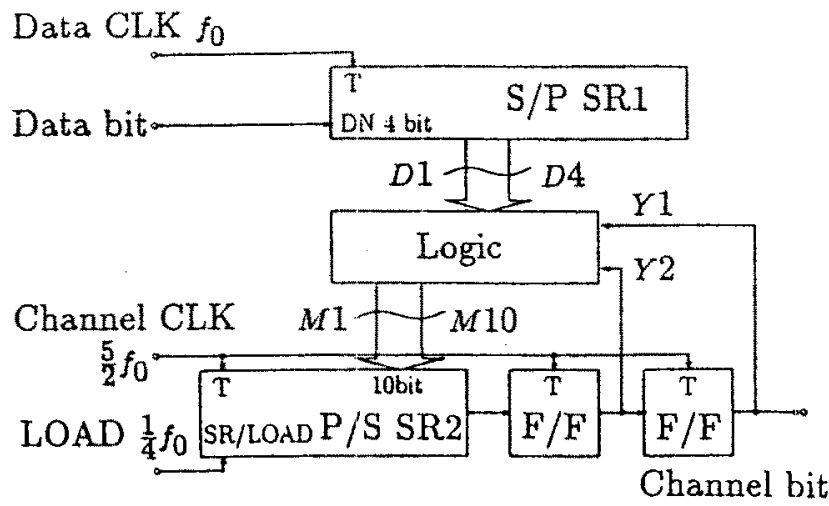

Fig.2:Encoding circuit of $(2,5)$ RLL code

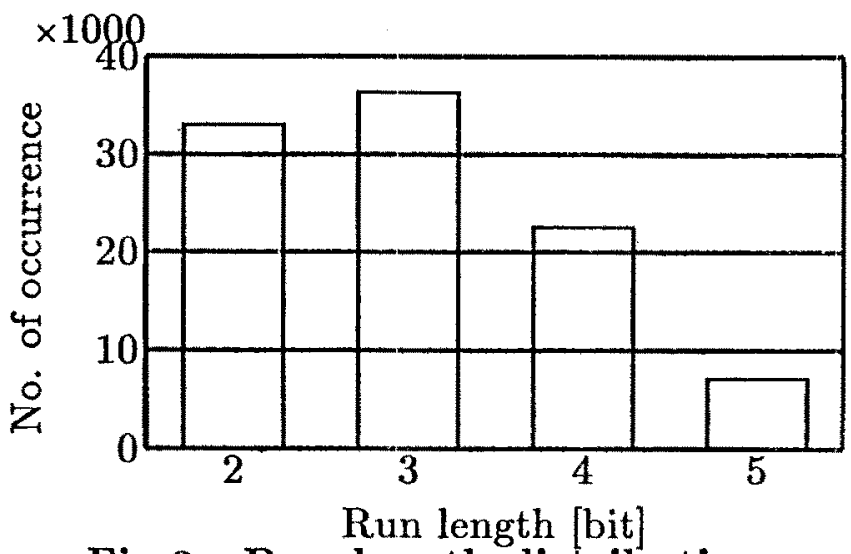

Fig.3 : Run length distribution

occurrence probability of run length of 3,4 are large, the code has good recording and play back characteristics. Fig. 4 illustrates error propagation characteristics when one bit

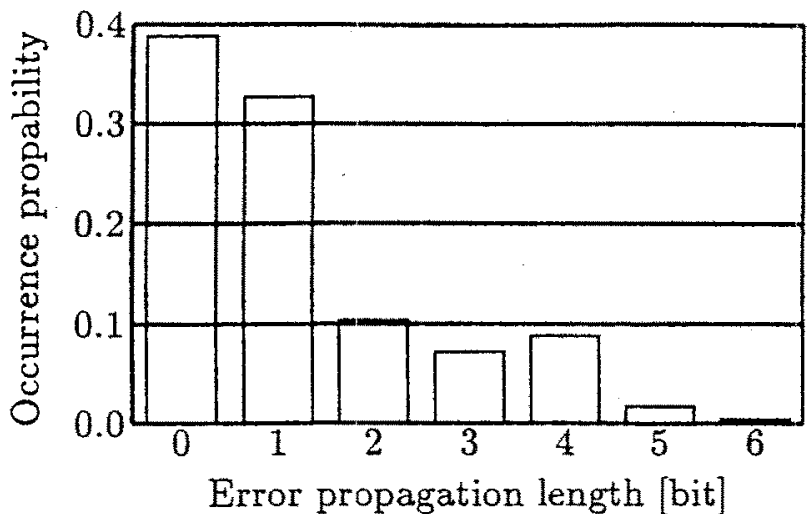

Fig.4: Error propagation characteristics shift takes place in play back signal. The maximum and average propagation are less than $6 \mathrm{~T}$ and $1.2 T$ respectively so that burden to the EDAC is small.

\section{Groove Parameter}

The worst case is repetition of 5T,3T pattern so that the pit density is 0.6 . The groove parameter can be dramatically improved. Fig. 5 and 6 show computer simulated pattern of pit and space pattern of $(1,7)$ RLL code and that of $(2,5)$ RLL code. The black part corresponds to the pit. The picture shows pattern in the middle of the zone of zone Constant Angular Velocity (ZCAV) so the edge of the zone is not shown. There is re-synch. byte which controls DSV in the meddle of each tack. Therefore, left part of the picture is the worst combination while right half is the best combination. Small pits are homogeneously distributed on the track in $(2,5)$ RLL. This is the cause of good groove signal.

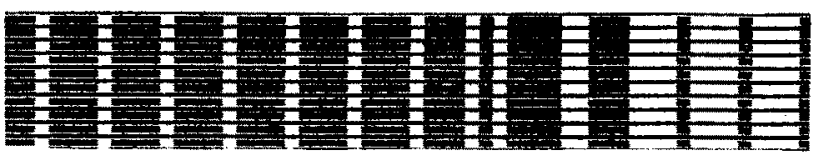

Fig.5: The worst case pit pattern of $(1,7)$ RLL code

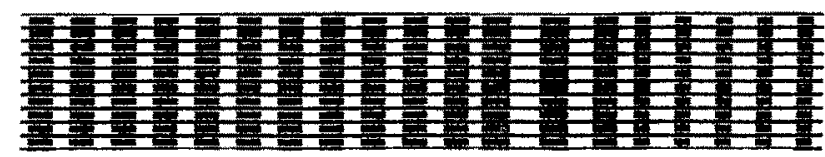

Fig.6: The worst case pit pattern of $(2,5)$ RLL code

\section{Window Margin}

As seen from Table 2 Tmin and Detection window width (Tw) of $(2,5)$ RLL code are shorter than that of $(1,7)$ RLL code. This comes from smaliness of Shannon capacity of $(2,5) \mathrm{RLL}$ code.[2] When $(2,5) R L L$ code is used in 
prepit area the recording density shall not be smaller than that when $(1,7)$ RLL code is recorded in MO area. The narrow band signal to noise ratio (NBSNR) of pre-pit signal is better than that of MO signal. This largeness of NBSNR saves the smallness of the capacity of $(2,5)$ RLL code. The window margin of $(2,5)$ RLL when it is recorded in pre-pit area and that of $(1,7)$ RLL code recorded in MO area were compared. The standard deviation of jitter as a percentage of detection window width of both $(2,5)$ RLL code and that of $(1,7)$ are listed in Table 3. The jitter of $(1,7) \mathrm{RLL}$ was experimental value. The jitter caused by noise of $(2,5)$ RLL code was calculated from NBSNR, and that caused by Inter-Symbol Interference (ISI) of $(2,5)$ RLL pre-pit recording was obtained by computer simulation. The indicial response was calculated by program computing optical diffraction at pit area. The indicial responses were added according to the evaluation pit pattern. The pit pattern were $8 * 3 \mathrm{~T}, 6 \mathrm{~T}, 3 \mathrm{~T}, 6 \mathrm{~T}, 3 \mathrm{~T}, 3 \mathrm{~T}, 6 \mathrm{~T}, 3 \mathrm{~T}$, $6 \mathrm{~T}, 3 \mathrm{~T}$, where $8 * 3 \mathrm{~T}$ means eight times repetition of 3T interval. The read out signal was processed by the direct slicing method, and the jitter was measured. [3]

The optical condition for comparison were same for both code. That is wave length of the laser beam was $780 \mathrm{~nm}$, NA of the object lens was 0.55, and recorded in $1773 \mathrm{bit} / \mathrm{mm}$.

Table3: Jitter of $(2,5)$ RLL and $(1,7)$ RLL code caused by noise and ISI

\begin{tabular}{|c|c|c|}
\hline Code & Noise & IST+Noise \\
\hline$(2,5)$ RLL & $2.8 \%$ & $5.9 \%$ \\
\hline$(1,7)$ RLLL & $4.9 \%$ & $5.3 \%$ \\
\hline
\end{tabular}

\section{CONCLUSION}

In order to improve groove parameter at ROM area with mark edge recording, new $(2,5)$ RLL code whose Tmax is small were developed. Improvement of groove parameter was achieved.

\section{ACKNOWLEDGEMENT}

The authors wish to express thank to MCC to supply data of CTS shown in Figure 1.

\section{REFERENCE}

[1] ISO/IEC DIS-13842, 1994.

[2] K. A. S. Immink "Coding Techniques for Digital Recorders," Prentice Hall, 1991.

[3] T. Maeda et al. "Read Channel and Format for High Density Magneto-Optical Disk System, Th2.3 ISOM/ODS'93, PP. 59-60, 1993.2

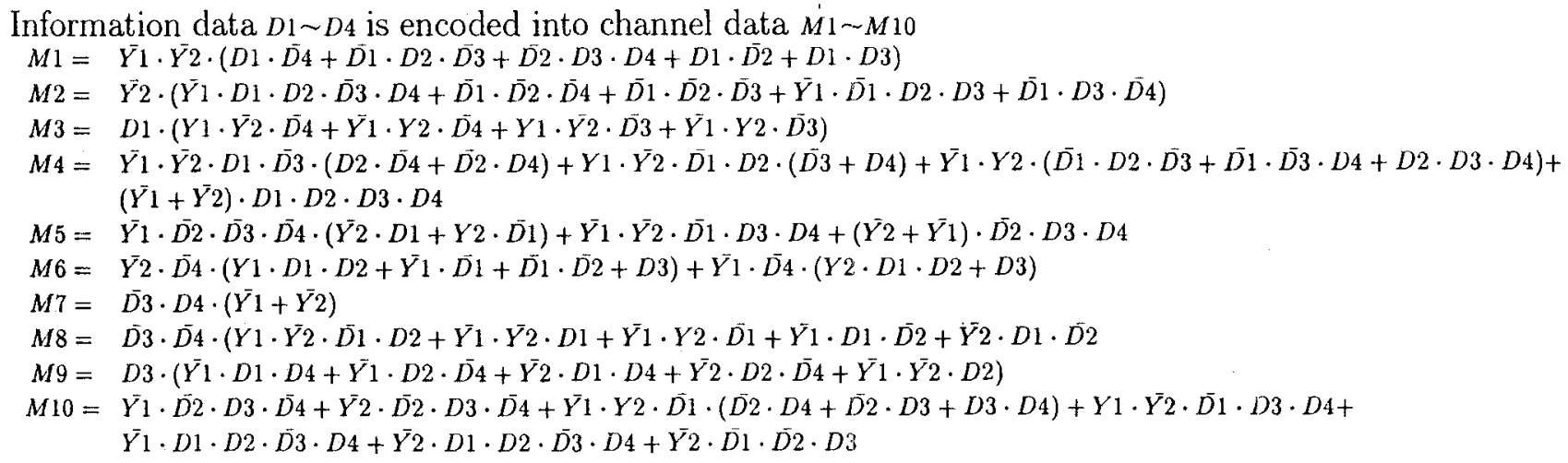

\section{Annex1:Encoding rule of $(2,5)$ RLL code}

